\title{
Ruptured Haemorrhagic Corpus Luteal Cyst- A Gynaecologist's Nightmare - Case Report
}

\section{Priya Pratapan Nair ${ }^{1}$, Amruta Chaudhary ${ }^{2}$, Arpita Jaiswal ${ }^{3}$}

Section: Healthcare

Sci. Journal Impact

Factor: 6.1 (2018)

ICV: 90.90 (2018)

cc) (i) (9)

Copyright@IJCRR
'Assistant Professor, Department of OBGY, Datta Meghe Medical College and Shalinitai Meghe Hospital and Research Centre, Wanadongri, Hingna, Nagpur- 441110, India; 'Assistant Professor, Department of OBGY, Datta Meghe Medical College and Shalinitai Meghe Hospital and Research Centre, Wanadongri, Hingna, Nagpur-441110, India; 3Professor Department of OBGY Jawaharlal Nehru Medical College, Datta Meghe Institute of Medical Sciences, Sawangi, Wardha-442001, India.

\section{ABSTRACT}

Women in the reproductive age group presenting in an emergency with acute pelvic pain is a frequent gynaecological condition. Various differential diagnoses including non-gynaecological causes should be kept in mind while assessing the case. Most common gynaecological causes of acute pelvic pain include ruptured ectopic pregnancy and ruptured haemorrhagic corpus luteal cyst. Meticulous history taking, clinical examination, and laboratory workup is required to confirm the diagnosis and timely intervention is needed to reduce maternal morbidity and mortality. Hereby, I would discuss a case of spontaneous rupture of haemorrhagic corpus luteal cyst in a post mitral valve replaced patient on an oral anticoagulant.

Case: A 22-year-old woman with 3 abortions, no live issues, known case of Rheumatic Heart Disease (Mitral Stenosis), Mitral Valve Replacement done in 2012, the patient was on oral warfarin, presented in casualty with acute pain in the lower abdomen, nausea and vomiting with tachycardia with no history of amenorrhoea.

Diagnosis: Initial Blood investigations were within normal limits and transabdominal ultrasonography was suggestive of right ovarian tumour with benign neoplastic etiology.

Intervention: Initially the patient was managed conservatively, later her condition deteriorated with tachycardia and hypotension with free fluid in the abdomen when she was taken for emergency exploratory laparotomy with transfusion of required blood products keeping in mind the oral anticoagulant and not to overload the patient. Persistent tachycardia in the postoperative period despite adequate replacements leads to her evaluation of thyroid profile when she was diagnosed as hyperthyroidism and was started on medications when her condition improved tremendously. The patient recovered well post-op.

Conclusion: The patient with acute abdomen should be evaluated for all the differential diagnoses, primarily excluding pregnancy and other non gynaecological conditions. Timely diagnosis and management can save a patient's life.

Key Words: Corpus luteal cyst, Rheumatic heart disease, Mitral stenosis, Mitral valve replacement, Warfarin, Exploratory laparotomy

\section{INTRODUCTION}

Acute pain is a common entity with which women of all groups can present in emergency ${ }^{1}$. It is rapid in onset, associated with unstable vitals and obvious abnormalities in physical examination and laboratory assessment. A timely and thorough examination, guided by each organ system evaluation of pathology will ensure effective diagnosis and management. ${ }^{2,3}$ Causes of acute pain in the reproductive age group include ectopic pregnancy, inevitable abortion, endometritis, pelvic inflammatory disease, tubo ovarian abscess, haemorrhagic corpus luteal cyst 4 . Symptoms may overlap with other gastrointestinal and urinary tract causes like ap- pendicitis, cholecystitis, ureteric colic, etc. ${ }^{5}$ In the evaluation of acute pelvic pain, early diagnosis is critical to reducing maternal morbidity and mortality. History of amenorrhoea, nausea, vomiting, abdominal distention, constipation, fever with chills and symptoms of haemoperitoneum like orthostasis, right upper quadrant pain, shoulder pain should be meticulously asked for. ${ }^{1}$

Spontaneous massive haemoperitoneum due to rupture of haemorrhagic corpus luteal cyst in a Mitral Valve Replaced patient on oral anticoagulant is a rare entity and only a few cases have been reported. Due to the effect of anticoagulant, haemorrhage was added effect requiring vitamin $\mathrm{K}$, blood

\section{Corresponding Author:}

Dr. Priya Pratapan Nair, Assistant Professor, Department of OBGY, Datta Meghe Medical College and Shalinitai Meghe Hospital and Research Centre, Wanadongri, Hingna, Nagpur, India; Email: priyapratapan@gmail.com

ISSN: 2231-2196 (Print)

Received: 16.06 .2020
ISSN: 0975-5241 (Online)

Revised: 18.07.2020

Accepted: 06.08.2020
Published: 22.08 .2020 
products, and fresh frozen plasma to wear off the effect of warfarin. Hereby, presenting the case of a nulliparous woman who presented in emergency with acute abdomen due to ruptured haemorrhagic cyst who was managed efficiently with emergency exploratory laparotomy and adequate blood products keeping in mind the preexisting treated heart disease.

\section{CASE SUMMARY}

The patient, 22 years old, resident of Amravati was admitted via casualty with complaints of pain in the abdomen (mild-moderate) since 1 month (increased to severe, since noon), nausea \& vomiting (on \& off) since 1 month. Last menstrual period- 19/4/20. Last to last menstrual period23/3/20, Regular, 25-30 days cycles, Average flow. Obstetric History: Married for 3 yrs. A3 - 3 Spontaneous abortions at 2-2 $\mathrm{n}$ half month gestation, (dilatation and evacuation done). On Admission: General condition was moderate, thin built, afebrile, pallor present, pulse- $120 / \mathrm{min}$, no Icterus, exophthalmos present, $\mathrm{BP}=110 / 70 \mathrm{~mm} \mathrm{Hg}$. On systemic examination: respiratory system was normal, cardiovascular system - Tachycardia present, Mechanical valve click present, Per abdomen: Soft, mild tenderness present on (left iliac fossa), Per speculum: Cervix, Vagina -Healthy, min bleeding present through cervical os, on per vaginal examination: Uterus- normal-sized, anteverted, left Fornix-fullness present, tender ness present, right Fornix- free, non-tender.

Patients History: Patients was operated outside for rightsided twisted ovarian cyst on 31/12/19 right Salpingo-oophorectomy was done. Again, was operated on 15 April'2020 - Left Salpingectomy for pain in abdomen with USG S/O Serous Cyst Adenoma. K/C/O Heart disease (RHD with MS) Mitral Valve replacement done in 2016, since then, pt was on T. Warfarin 5mg OD. Patients ultra-sonography on 30/4/20 was suggestive of well defined solid cystic lesion in left adnexa of $7.9 \times 7.6 \times 8 \mathrm{~cm}$, with thin septations and no internal vascularity or calcification. Right ovary not separately visualised. Suggestive of benign neoplastic etiology.

Investigations: International normalized ratio was 2.07, Haemoglobin was $11.1 \mathrm{gm} \%$, platelet was $1,95,000$, partial thromboplastin time was $46 \mathrm{Sec}$. In Liver function test total bilirubin was 1.3, SGOTwas 10, SGPT was 62 . Kidney function test - blood urea- 30 , serum creatinine- 0.6 , serum sodium - 135, serum potassium- 4.1, sickling negative, Urine pregnancy test was negative. Due to persistent tachycardia (pulse - 120-130/min) her physician opinion was done, and she was started on metXL 25 once daily and Laci lactoneonce daily. ECG was s/o sinus tachycardia. $2 \mathrm{D}$ echo done was normal. Initially, she was conservatively managed in view of the benign cyst but the patient's condition deteriorated with persistent tachycardia and hypotension developed so her tap- ping was done s/o haemoperitoneum. She was explored immediately on $30 / 4 / 20$

Intraoperative evidence of heamoperitoneum of $1500 \mathrm{cc}$ with well-organized clots and ruptured cyst of left ovary. Uterus identified- normal. Right ovary and tube not visualized. Leftsided cyst wall adherent to the posterior surface of the uterus and surrounding intestine. The cyst wall separated by blunt and sharp dissection. As the patient was on oral warfarin, preop inj. Vitamin K, 2 fresh frozen plasma, and 1 whole blood given. Intra-op 2 whole blood, 2 fresh frozen plasma, and 1 platelet transfusion given. Post-op 2 whole blood given. Drain kept in the pouch of Douglas.

Post-operative events: Post-op day 1 - ECG s/o global ischemia. Troponin T-neg. (Creatinine phosphokinase MB) CPKMB- 52.8 IU/ML. The patient was started on t. atorvas and acitron. Drain output $600 \mathrm{ml}$ in $24 \mathrm{hrs}$.

Post-op day 2- the patient was started on injectable heparin four time a day dose as suggested by a cardiologist with daily International normalised ratio. Daily International normalised ratio was done in the range of $2-2.3$. Post-op day 8- drain output was few cc in 24 hrs so drain was removed. Pt's International normalised ratio was 2 so she was started on heparin four times a day with warfarin $5 \mathrm{mg}$ once a day. Post-op day 11- pt had tachycardia and palpitation with intermittent gastroenteritis, managed conservatively. Postop day 17- International normalised ratio was 2 . She was shifted to oral warfarin. Due to persistent tachycardia and palpitation on Post-op day 18- thyroid profile sent s/o overt hyperthyroidism. Serum T3- 429.9 ng/ d1. Serum T4- 24.8 $\mathrm{ug} / \mathrm{dl}$. TSH- $<0.01 \mathrm{iu} / \mathrm{ml}$. She was started on t. metxl $25 \mathrm{mg}$ once a day with t. neomercazole $10 \mathrm{mg}$ thrice a day and t. atorvas and acitrom were continued. Gradually tachycardia settled, the general condition improved. Histopath report was s/o haemorrhagic corpus luteal cyst.

\section{DISCUSSION}

Functional ovarian cysts (follicular and corpus luteal cysts) are most the common ovarian cysts and are more likely to rupture than benign or malignant neoplasms. During ovulation, a small amount of blood leaks into the peritoneal cavity and a high concentration of fluid prostaglandins causes midcycle pain (mittelschmerz). ${ }^{6}$ Pain is usually mild to moderate and self-limited, with an intact coagulation system, haemoperitonem is unlikely. The corpus luteum is formed during the ovarian cycle's luteal phase, and supports the growth of the pregnancy. The central cavity of maturing corpus luteum is filled with blood by the spontaneous but limited bleed. Blood is consumed and the tiny cystic space sequence is created. ${ }^{7}$ The corpus luteum enlarges when hemorrhage becomes severe, and often forms a large cystic structure. A ruptured corpus luteum doesn't cause excessive bleeding most of the time. 
However catastrophic blood loss can be, requiring surgical intervention and transfusion of blood. Rupture and resulting bleeding are frequently caused by exercise, coitus, trauma, or a pelvic exam. ${ }^{8}$ Haemorrhagic corpus luteal cyst is the most common cyst to rupture and cause haemoperitoneum. Other uncommon causes are uterine rupture, endometriosis, and rupture hydrosalphinx. ${ }^{5}$ The patient may present with a wide range of clinical signs with the majority having no signs of severe peritoneal irritation. Evaluation of serum beta hCG helps to differentiate between ruptured ectopic pregnancy and cyst. ${ }^{4,9}$ as symptoms of ruptured corpus luteal cysts are the same as ruptured ectopic. Initial blood investigations along with trans vaginal ultrasonography are helpful to diagnose the cause.

Doppler ultrasonography helps to identify the vascularity and type and nature of fluid and also the cyst wall., ${ }^{40} \mathrm{~A}$ contrast $\mathrm{CT}$ scan has more role in diagnosing a corpus luteal cyst as the wall appears thickened and shows CT enhancement post dye due to increased vascularity. ${ }^{4,10}$ MRI may not be of help in cases of corpus luteal cysts as compared to CT scan. ${ }^{11}$ Treatment targets at preserving ovarian function and eliminating the cause of bleeding. ${ }^{12}$ Surgical exploration is indicated if there is significant haemoperitoneum or chemical peritonitis (endometrioma, dermoid) which can be lifethreatening.

In patients with artificial heart valves, long-term anticoagulation is required to prevent thromboembolic complications. In such cases, an international standardized ratio (INR) target for anticoagulation must be preserved to prevent thromboembolism, which must be carefully controlled to avoid haemorrhagic complications due to the narrow therapeutic index of vitamin $\mathrm{K}$ antagonists. ${ }^{13,14}$. There are much debate and confusion about the type of treatment needed to reverse anticoagulation for emergency surgery and the optimal time to safely restart anticoagulation therapy. ${ }^{13,14,15}$

Spontaneous bleeding is one of the warfarin's most common adverse effects, and factors such as age, dosage, length of treatment, drug exposure, and occult diseases further determine the risk of bleeding. ${ }^{16}$ Strict monitoring of the coagulation profile is important in patients who take longterm warfarin. Bleeding and haemorrhage often linked to a ruptured corpus luteum cyst may occur in women in the reproductive age group. Haemoperitoneum is an unusual and often frightening situation to find in these anticoagulant patients. ${ }^{14}$ Such patients pose a challenge as abrupt anticoagulation reversal is needed to manage any ongoing haemorrhage and further blood loss from surgery. ${ }^{15}$.

There are typically three choices for an immediate anticoagulant reversal in patients receiving the vitamin $\mathrm{K}$ antagonist: vitamin $\mathrm{K}$, prothrombinase complex concentrate (PCC), and Fresh frozen plasma (FFP). FFP can be used in patients needing volume restauration and warfarin reversal if available and pre-thawed immediately. ${ }^{16}$ In patients with mechanical mitral valves, FFP alone or in combination with low dose vitamin $\mathrm{K}$ (1-2 mg intravenous) is preferable..$^{13,17}$

According to Hallatt et al. ${ }^{18}$, the hemorrhage of a ruptured corpus luteum cyst is likely to be smaller than that of ectopic pregnancy and is likely to be non-recurring until it ends. Corpus luteal cysts are generally reported on pelvic ultrasonic studies as incidental structures. Corpus luteum cyst rupture with intra-abdominal hemorrhage can appear ultrasonographically similar to a ruptured ectopic pregnancy, as a negative serum pregnancy test can be a discerning feature in our case. ${ }^{18,19}$ For one analysis, instead of the cyst itself, the hemoperitoneum was found to be the dominant imagery feature. Hemoperitoneum from a ruptured hemorrhagic ovarian cyst displays imagery-like characteristics from other sources, such as ruptured ectopic pregnancy. ${ }^{20}$ The diagnosis is based mainly on elevated clinical skepticism, laboratory evidence, and results from ultrasounds. Historically the ectopic pregnancy is the main differential diagnosis. Coincidental involvement of corpus luteum cyst rupture has also been recorded with ectopic pregnancy. ${ }^{19,21,22}$ Therefore, even when intrauterine or extrauterine pregnancy is confirmed, the probability and incidence of corpus luteum cyst rupture should be kept in mind. Corpus luteum haemorrhage treatment is cautious or chirurgical. ${ }^{14,23,24}$ This may depend on the patient's haemodynamic state and the volume of haemoperitoneum in during clinical evaluation and ultrasonography.

Hormone contraception may be used to avoid ovarian cysts, resulting in a possible reduction in the associated morbidity and mortality of cystic hemorrhage during anticoagulation. Implementing safe and successful ovulation suppression in adults with CHD remains challenging given their underlying hypercoagulable state and difficulties in predicting risk for the development and rupture of hemorrhagic cysts. ${ }^{25} \mathrm{De}-$ spite their limited safety evidence, the European Cardiological Society is currently advising against the use of combined oral contraceptives. In particular, caution should be extended to preparations containing estrogen in patients with unrepaired defects; those with surgically corrected defects with associated mechanical prostheses; and those with cyanosis, ventricular dysfunction, atrial fibrillation or Eisenmenger physiology complicated defects. ${ }^{26}$ On the other hand, progestin-only formulations, including depomedroxyprogesterone acetate (DMPA) and desogestrel, can be used safely in adults with CHD undergoing anticoagulation care, but with variable efficacy in ovulation suppression. ${ }^{27}$ Certain related articles from this region are available. ${ }^{28-37}$

\section{CONCLUSION}

Any women of reproductive age group presenting in an emergency with acute abdomen should be ruled out for 
gynaecological and non gynaecological causes meticulously for proper diagnosis and timely management. Patients who are on oral anticoagulants have more chances of bleeding tendencies should be properly given antagonists to prevent excessive blood loss in case of managing the patients surgically. Routine INR follow up should be done to maintain its level. Patient with persistant tachycardia, despite all the appropriate management, can be misleading and they should be searched for other underlying causes like sepsis or overt hyperthyroidism. Prompt diagnosis and management are required to decrease the significant patient morbidity and mortality.

\section{Acknowledgement: None}

\section{Conflict of interest: None}

\section{Source of Funding: None}

\section{REFERENCES}

1. G. P. A. Samraj and R. W. Curry Jr., "Acute pelvic pain: evaluation and management," Comprehensive Therapy, vol. 30, no. 3, pp. 173-184, 2004. View at: Google Scholar

2. A. Potter and C. Chandrasekhar, "US and CT evaluation of acute pelvic pain of gynecologic origin in nonpregnant premenopausal patients," Radiographics, vol. 28, no. 6, pp. 1645-1659, 2008. View at: Publisher Site | Google Scholar

3. H. Kayaba, H. Tamura, K. Shirayama, J. Murata, and Y. Fujiwara, "Hemorrhagic ovarian cyst in childhood: a case report," Journal of Pediatric Surgery, vol. 31, no. 7, pp. 978-979, 1996. View at: Publisher Site | Google Scholar

4. O. Roche, N. Chavan, J. Aquilina, and A. Rockall, "Radiological appearances of gynaecological emergencies," Insights into Imaging, vol. 3, no. 3, pp. 265-275, 2012.

5. B. Coulier, S. Malbecq, P. E. Brinon, and A. Ramboux, "MDCT diagnosis of ruptured tubal pregnancy with massive hemoperitoneum," Emergency Radiology, vol. 15, no. 3, pp. 179-182, 2008. View at: Publisher Site | Google Scholar

6. Swann RT, Bruce NW (1987) Oxygen consumption, carbon dioxide production and progestagen secretion in the intact ovary of the Day-16 pregnant rat. J ReprodFertil 80(2): 599-605.

7. Tang LC, Cho HK, Chan SY, Wong VC (1985) Dextropreponderance of corpus luteum rupture. A clinical study. J Reprod Med 30(10): 764-768

8. Stenchever M. Comphrehensive gynaecology, $5^{\text {th }}$ ed. USA, Mosby;2007:460-1.

9. Y. Kaakaji, H. V. Nghiem, C. Nodell, and T. C. Winter, "Sonography of obstetric and gynecologic emergencies: part II, gynecologic emergencies," American Journal of Roentgenology, vol. 174, no. 3, pp. 651-656, 2000.View at: Google Scholar

10. L. Valentin, "Use of morphology to characterize and manage common adnexal masses," Best Practice \& Research Clinical Obstetrics \&Gynaecology, vol. 18, no. 1, pp. 71-89, 2004.View at: Google Scholar

11. F. Miller, R. Bree, M. Rosen et al., "ACR Appropriateness Criteria," American College of Radiology, 2008, http://www.acr.org/. View at: Google Scholar

12. N. Gupta, V. Dadhwal, D. Deka, S. K. Jain, and S. Mittal, "Corpus luteum hemorrhage: rare complication of congenital and acquired coagulation abnormalities," The Journal of Obstetrics and Gynaecology Research, vol. 33, no. 3, pp. 376-380, 2007.

13. Panduranga $\mathrm{P}$, Al-Mukhaini $\mathrm{M}$, Al-Muslahi $\mathrm{M}$, Haque MA, Shehab A. Management dilemmas in patients with mechanical heart valves and warfarin-induced major bleeding. World J Cardiol2012;4:54-9.

14. Jamal A, Mesdaghinia S. Ruptured corpus luteum cysts and anticoagulant therapy. Int J GynaecolObstet2002;76:319-20.

15. Curtis R, Schweitzer A, van Vlymen J. Reversal of warfarin anticoagulation for urgent surgical procedures. Can J Anesth2015;62:634-49.

16. Hunt B, Levi M. Urgent reversal of vitamin $\mathrm{K}$ antagonists. BMJ 2018;360:j5424.

17. Garcia DA, Crowther MA. Reversal of warfarin: Case-based practice recommendations. Circulation 2012;125:2944-7.

18. Hallatt JG, Steele CH, Snyder M (1984) Ruptured corpus luteum with hemoperitoneum: a study of 173 surgical cases. Am J ObstetGynecol 149(1): 5-9.

19. Yoffe N, Bronshtein M, Brandes J, Blumenfeld Z (1991) Hemorrhagic ovarian cyst detection by transvaginalsonography: The great imitator. Gynecol Endocrino 15(2): 123-129.

20. Hertzberg BS, Kliewer MA, Paulson EK (1999) Ovarian cyst rupture causing hemoperitoneum: imaging features and the potential for misdiagnosis. Abdom Imaging 24(3): 304-308.

21. Raziel A, Ron-El R, Pansky M, Arieli S, Bukovsky I, et al. (1993) Current management of ruptured corpus luteum. Eur J Obstet Gynecol Reprod Biol 50(1): 77-81.

22. Jeffrey RB, Laing FC (1982) Echogenic clot: a useful sign of pelvic hemoperitoneum. Radiology 145(1): 139-141.

23. Hoffman R, Brenner B (2009) Corpus luteum hemorrhage in women with bleeding disorders. Women's Health (Lond) 5(1): 91-95.

24. Payne JH, Maclean RM, Hampton KK, Baxter AJ, Makris M (2007) Haemoperitoneum associated with ovulation in women with bleeding disorders: the case for conservative management and the role of the contraceptive pill. Haemophilia 13(1): 93-97.

25. Canobbio M.M. (2004) Contraception for the adolescent and young adult with congenital heart disease. Nurs Clin North Am 39:769-785.

26. Baumgartner H., Bonhoeffer P., De Groot N.M.,et al. (2010) ESC guidelines for the management of grown-up congenital heart disease. Eur Heart J 31:2915-2957.

27. Milsom I.,Korver (2008) Ovulation incidence with oral contraceptives: a literature review. J Fam Plann Reprod Health Care 34-237.

28. Bhriegu, R., M. Agrawal, and C. Hariharan. "Assessment of Maternal and Perinatal Outcome in Postdated Pregnancy." Journal of Datta Meghe Institute of Medical Sciences University 12, no. 1 (2017): 35-40. https://doi.org/10.4103/jdmimsu.jdmimsu_20_17.

29. Gaikwad, K.B., N.G. Joshi, and S.P. Selkar. "Study of Nitrosative Stress in Pregnancy Induced Hypertension." Journal of Clinical and Diagnostic Research 11, no. 3 (2017): BC06-8. https://doi.org/10.7860/JCDR/2017/23960.9396.

30. Roy, M., U.L. Gajbe, B.R. Singh, and P. Thute. "Morphometric Measurement of Fetal Femur Length for the Prediction of Gestational Age in the Ii Nd and Iii Rd Trimester of Pregnancy by Ultrasonography." Journal of Datta Meghe Institute of Medical Sciences University 12, no. 3 (2017): 187-90. https://doi. org/10.4103/jdmimsu.jdmimsu_71_17.

31. Patil, S., R. Ranka, M. Chaudhary, A. Hande, and P. Sharma. "Prevalence of Dental Caries and Gingivitis among Pregnant and Nonpregnant Women." Journal of Datta Meghe Institute of Medical Sciences University 13, no. 1 (2018): 44-47. https:// doi.org/10.4103/jdmimsu.jdmimsu_5_18. 
32. Yadav, S., M. Agrawal, C. Hariharan, D. Dewani, K. Vadera, and N. Krishna. "A Comparative Study of Serum Lipid Profile of Women with Preeclampsia and Normotensive Pregnancy." Journal of Datta Meghe Institute of Medical Sciences University 13, no. 2 (2018): 83-86. https://doi.org/10.4103/jdmimsu. jdmimsu 7017.

33. Phatak, S., D. Shrivastav, G. Marfani, S. Daga, K. Madurwar, and S. Samad. "Transvaginal Sonography and Elastography Evaluation of Ectopic Pregnancy." Journal of Datta Meghe Institute of Medical Sciences University 14, no. 2 (2019): 86-89. https://doi.org/10.4103/jdmimsu.jdmimsu_13_19.

34. Widmer, M., G. Piaggio, T.M.H. Nguyen, A. Osoti, O.O. Owa, S. Misra, A. Coomarasamy, et al. "Heat-Stable Carbetocin Versus Oxytocin to Prevent Hemorrhage after Vaginal Birth."
Obstetrical and Gynecological Survey 73, no. 11 (2018): 613-14. https://doi.org/10.1097/OGX.0000000000000616.

35. Yeola, M.E., D. Gode, and A.K. Bora. "Diagnostic Laparoscopy as an Effective Tool in Evaluation of Intra-Abdominal Malignancies." World Journal of Laparoscopic Surgery 11, no. 2 (2018): 68-75. https://doi.org/10.5005/jp-journals-10033-1338.

36. "Evaluation of Abdominal Malignancies by Minimal Access Surgery: Our Experience in a Rural Setup in Central India." World Journal of Laparoscopic Surgery 11, no. 3 (2018): 11520. https://doi.org/10.5005/jp-journals-10033-1350.

37. Anamul Haq, Sheema, Shazieya Akhtar. Etiology of Abnormal Uterine Bleeding in Adolescents - Emphasis Upon Polycystic Ovarian Syndrome International Journal of Current Research and Review. Vol 12 Issue 10, May, 05-09 